\title{
Syntheses of Phenylthio- and Phenyl-5-Nitro-2-Furoates, and Their Antibacterial Activities
}

\author{
Eiji NIwa, Hajimu TANABE and Masato MIYAKE* \\ (Received November 22, 1968)
}

The establishment of 5-nitro-2-furfural semicarbazone as a drug of the synthetic type has introduced mass-researches finding new derivatives of this type, in which typical examples are known as 5-nitro-2-furan-acrylamide and more recently as 2-(2-furyl)-3-(5-nitro2 -furyl)-acrylamide, and these have been used in medical and other field.

However, as well known, these are rather labile as a food preservatives; for example, the heating at $85^{\circ} \mathrm{C}$ for sixty minutes in manufacturing of fish sausage causes break down of one-half of them into ineffective substance to micro-organisms. ${ }^{1}$

So, it is desirable to prepare a new effective and more stable derivative from chemotherapic as well as other practical standpoint.

On the other hand, in the course of synthetic study of furan compounds, found one of the authors that phenylthio-2-furoates showed strong inhibitive action on Trichophyton asteroides. ${ }^{2)}$

Now, the series of derivatives, phenylthio-5-nitro-2-furoates analogous to phenylthio2-furoates mentioned above and phenyl-5-nitro-2-furoates, were prepared for the study of antibacterial activities.

The latters were synthesized with the view to comparative study as to biological activities to the formers.

\section{Experimental}

Preparation of phenylthio-5-nitro-2-furoates (IV) and phenyl-5-nitro-2-furoates (V).

The phenylthio-5-nitro-2-furoates (IV) and phenyl-5-nitro-2-furoates (V) were prepared as shown in schematic diagram, Fig. 1.

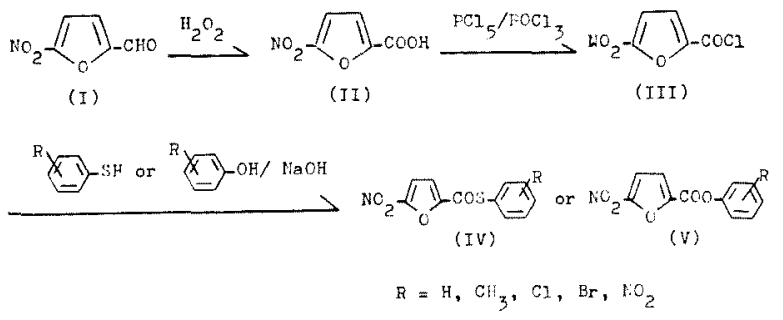

Fig. 1. Schematic diagram of phenylthio-5-nitro-2-furoates (IV) and phenyl-5-nitro-2-furoates (V).

* Faculty of Fisheries, Prefectural University of Mie, Tus, Japan (丹羽栄二, 田辺一, 三宅正人：三重県 立大学水産学部) 
5-Nitro-2-furoic acid (II); (II) is obtained by oxidation of 5-nitro-2-furfural (I) with hydogen peroxide in pyridine. ${ }^{3)}$

5-Nitro-2-furoyl chloride (III); To a suspension of $10 \mathrm{~g}$ (II) of $20 \mathrm{ml}$ phosphorous oxychloride in a $200 \mathrm{ml}$ round bottomed flask fitted with reflux condenser, is added slowly $15 \mathrm{~g}$ phosphorous pentachloride under gently boiling. A tube from the top of the condenser conducts hydrogen chloride to cold water.

The addition of the phosphorous pentachloride occupies about one hour, and then heating is continued for two hours. The mixture is distillated under reduced pressure. The crude product boiling at $112-5^{\circ} \mathrm{C} / 15 \mathrm{mmHg}$ is collected. To this distillate is added $100 \mathrm{ml}$ of ice-water, then white precipitate is collected by sucktion and dried.

The crude product thus obtained is sufficient for following purposes. Yield $6.0 \mathrm{~g}$ $(53.6 \%)$

Phenylthio- and phenyl-5-nitro-2-furoates, (IV and V); In a $50 \mathrm{ml}$ round bottomed flask fitted with a reflux condenser, is placed a suspension of $0.9 \mathrm{~g}$ (III) and $10 \mathrm{ml}$ of $2 \%$ sodium hydroxide solution of corresponding thiophenol or phenol $(0.5 \mathrm{~m}$ mole $)$ which is shown in Table 1. The mixture is then heated and kept boiling. After one-half hour it is cooled and transfered to a separatory funnel and extracted two to three times with $50 \mathrm{ml}$ of chloroform. The combined chloroform extract is washed with $50 \mathrm{ml}$ of $5 \%$ sodium hydroxide solution, then distillated water.

Chloroform layer is dried with anhydrous sodium sulfate and chloroform is evaporated. The residue is crystallized from methanol/water.

Thioesters (IV-a, b, c, d, e and f) and esters ( V-a, b, c, d, e and f) thus prepared are shown in Table 1.

Table 1. Phenylthio-5-nitro-2-furoates (IV) and phenyl-5-nitro-2-furoates (V).

\begin{tabular}{|c|c|c|c|c|c|c|c|c|c|}
\hline \multirow{3}{*}{ No } & \multirow[t]{3}{*}{ Compounds } & \multirow[t]{3}{*}{$\begin{array}{l}\text { Starting } \\
\text { materials }\end{array}$} & \multirow{3}{*}{${ }^{\text {M.p. }}$. } & \multirow{3}{*}{$\underset{\%}{\text { Yield }}$} & \multirow{3}{*}{ Formula } & \multicolumn{4}{|c|}{ Elemenatry analysis } \\
\hline & & & & & & \multicolumn{2}{|c|}{ Calculated } & \multicolumn{2}{|c|}{ Found } \\
\hline & & & & & & $\mathrm{C}$ & $\mathbf{H}$ & $\mathrm{C}$ & $\mathrm{H}$ \\
\hline$I V-a$ & $X=\mathrm{SC}_{6} \mathrm{H}_{5}$ & $\mathrm{C}_{6} \mathrm{H}_{5} \mathrm{SH}$ & 148 & 26 & $\mathrm{C}_{11} \mathrm{H}_{7} \mathrm{NO}_{4} \mathrm{~S}$ & 53.01 & 2.83 & 53.51 & 3.10 \\
\hline$I V-b$ & $\mathrm{~S}\left(\mathrm{p}-\mathrm{CH}_{3} \mathrm{C}_{6} \mathrm{H}_{4}\right)$ & $\mathrm{p}-\mathrm{CH}_{3} \mathrm{C}_{6} \mathrm{H}_{4} \mathrm{SH}$ & 92 & 50 & $\mathrm{C}_{12} \mathrm{H}_{9} \mathrm{NO}_{4} \mathrm{~S}$ & 54.75 & 3.45 & 55.52 & 3.54 \\
\hline IV $-\mathrm{c}$ & $\mathrm{S}\left(\mathrm{p}-\mathrm{ClC}_{6} \mathrm{H}_{4}\right)$ & $\mathrm{p}-\mathrm{ClC}_{6} \mathrm{H}_{4} \mathrm{SH}$ & 118 & 52 & $\mathrm{C}_{11} \mathrm{H}_{6} \mathrm{NO}_{4} \mathrm{ClS}$ & 46.57 & 2.13 & 47.17 & 2.42 \\
\hline IV-d & $\mathrm{S}\left(\mathrm{p}-\mathrm{BrC}_{6} \mathrm{H}_{4}\right)$ & $\mathrm{p}-\mathrm{BrC}_{6} \mathrm{H}_{4} \mathrm{SH}$ & 115 & 28 & $\mathrm{C}_{11} \mathrm{H}_{6} \mathrm{NO}_{4} \mathrm{BrS}$ & 40.26 & 1.84 & 40.90 & 2.48 \\
\hline IV-e & $\mathrm{S}\left(\mathrm{p}-\mathrm{NO}_{2} \mathrm{C}_{6} \mathrm{H}_{4}\right)$ & $\mathrm{p}-\mathrm{NO}_{2} \mathrm{C}_{6} \mathrm{H}_{4} \mathrm{SH}$ & 180 & 15 & $\mathrm{C}_{11} \mathrm{H}_{6} \mathrm{~N}_{2} \mathrm{O}_{6} \mathrm{~S}$ & 44.90 & 2.05 & 44.86 & 2.43 \\
\hline IV-f & $\mathrm{SCH}_{2} \mathrm{C}_{6} \mathrm{H}_{5}$ & $\mathrm{C}_{6} \mathrm{H}_{5} \mathrm{CH}_{4} \mathrm{SH}$ & 97 & 40 & $\mathrm{C}_{12} \mathrm{H}_{9} \mathrm{NO}_{4} \mathrm{~S}$ & 54.75 & 3.45 & 55.42 & 3.54 \\
\hline$V-a$ & $\mathrm{OC}_{6} \mathrm{H}_{5}$ & $\mathrm{C}_{6} \mathrm{H}_{5} \mathrm{OH}$ & 114 & 28 & $\mathrm{C}_{11} \mathrm{H}_{7} \mathrm{NO}_{5}$ & 56.66 & 3.02 & 57.10 & 2.63 \\
\hline$V-b$ & $\mathrm{O}\left(\mathrm{p}-\mathrm{CH}_{3} \mathrm{C}_{6} \mathrm{H}_{5}\right)$ & $\mathrm{p}-\mathrm{CH}_{3} \mathrm{C}_{6} \mathrm{H}_{4} \mathrm{OH}$ & 113 & 61 & $\mathrm{C}_{12} \mathrm{H}_{9} \mathrm{NO}_{5}$ & 58.30 & 3.67 & 58.34 & 3.49 \\
\hline$V-c$ & $\mathrm{O}\left(\mathrm{O}-\mathrm{ClC}_{6} \mathrm{H}_{4}\right)$ & o- $\mathrm{ClC}_{6} \mathrm{H}_{4} \mathrm{OH}$ & 112 & 23 & $\mathrm{C}_{11} \mathrm{H}_{6} \mathrm{NO}_{5} \mathrm{Cl}$ & 49.37 & 2.26 & 49.84 & 2.71 \\
\hline$V-d$ & $\mathrm{O}\left(\mathrm{p}-\mathrm{ClC}_{6} \mathrm{H}_{4}\right)$ & $\mathrm{p}-\mathrm{ClC}_{6} \mathrm{H}_{4} \mathrm{OH}$ & 137 & 45 & " & $"$ & ", & 50.12 & 2.53 \\
\hline $\mathrm{V}-\mathrm{e}$ & $\mathrm{O}\left(\mathrm{p}-\mathrm{BrC}_{6} \mathrm{H}_{4}\right)$ & $\mathrm{p}-\mathrm{BrC}_{6} \mathrm{H}_{4} \mathrm{OHI}$ & 138 & 40 & $\mathrm{C}_{11} \mathrm{H}_{6} \mathrm{NO}_{5} \mathrm{Br}$ & 42.33 & 1.94 & 43.12 & 2.36 \\
\hline$V-f$ & $\mathrm{O}\left(\mathrm{p}-\mathrm{NO}_{2} \mathrm{C}_{6} \mathrm{H}_{4}\right)$ & $\mathrm{p}-\mathrm{NO}_{2} \mathrm{C}_{6} \mathrm{H}_{4} \mathrm{OH}$ & 197 & 17 & $\mathrm{C}_{11} \mathrm{H}_{6} \mathrm{~N}_{2} \mathrm{O}_{7}$ & 47.49 & 2.17 & 48.30 & 2.35 \\
\hline
\end{tabular}


Determination of minimum inhibitory concentration of phenylthio-5-nitro-2-furoates (IV) and phenyl-5-nitro-2-furoates (V).

A usual serial dilution method was employed to establish the minimum inhibitive concentration. A range of concentrations of the test compound was incorporated into nutrient broth which was then inoculated with an enterotoxigenic strain of Staphylococcus aureus $209 p$, a strain of Bacilus subtilis and a strain of Esherichia coli. Incubation was at $37^{\circ} \mathrm{C}$ for 2 and 4 days.

\section{Results}

The relative effectiveness of phenylthio- and phenyl-5-nitro-2-furoates against Staph. aureus 209p is shown in Table 2. p-Bromo-phenylthio-5-nitro-2-furoate (IV-d) is superior to control, 2-(2-furyl)-3-(5-nitro-2-furyl)-acrylamide against the test organism used. This tendency was observed not only at 2 days after incubation but also 4 days, at which time 2-(2-furyl)-3-(5-nitro-2-furyl)-acrylamide had lost more of its effectiveness. Also, pchlorophenylthio-5-nitro-2-furoate (IV-c) and benzylthio-5-nitro-2-furoate (IV-f) were effective.

Generally, phenylthio-5-nitro-2-furoates (IV) were more effective than phenyl-5-nitro2 -furoates (V) in the bacteriostatic point, and similar tendency was observed also in the action of phenylthio-2-furoates and phenyl-2-furoates against Trichophyton asterioides.

The data of bacteriostatic action against other strains tested were abbreviated for the less effectiveness.

Table 2. Minimum inhibitive concentration of phenylthio-5-nitro-2furoates (IV) and phenyl-5-nitro-2-furoates (V) for Staphylococcus aurets $209 p$ determined at 2 and 4 days incubation at $37^{\circ} \mathrm{C}$ in nutrient broth.

\begin{tabular}{|c|c|c|c|}
\hline \multirow{2}{*}{ No } & & \multicolumn{2}{|c|}{$\begin{array}{l}\text { Minimum inhibitive Concentration } \\
(\gamma)\end{array}$} \\
\hline & & 2 Days & 4 Days \\
\hline IV $-\mathbf{a}$ & $\mathrm{X}=\mathrm{SC}_{6} \mathrm{H}_{5}$ & 5 & 5 \\
\hline IV-b & $\mathrm{S}\left(\mathrm{p}-\mathrm{CH}_{3} \mathrm{C}_{6} \mathrm{H}_{4}\right)$ & 0.5 & 10 \\
\hline IV $-\mathrm{c}$ & $\mathrm{S}\left(\mathrm{p}-\mathrm{ClC}_{6} \mathrm{H}_{4}\right)$ & 0.5 & 0.5 \\
\hline IV $-\mathrm{d}$ & $\mathrm{S}\left(\mathrm{p}-\mathrm{BrC}_{6} \mathrm{H}_{4}\right)$ & 0.1 & 1 \\
\hline IV-e & $\mathrm{S}\left(\mathrm{p}-\mathrm{NO}_{2} \mathrm{C}_{6} \mathrm{H}_{4}\right)$ & 10 & - \\
\hline$I V-f$ & $\mathrm{SCH}_{2} \mathrm{C}_{6} \mathrm{H}_{4}$ & 0.5 & 5 \\
\hline $\mathrm{V}-\mathrm{a}$ & $\mathrm{OC}_{6} \mathrm{H}_{4}$ & 10 & - \\
\hline$V-b$ & $\mathrm{O}\left(\mathrm{p}-\mathrm{CH}_{3} \mathrm{C}_{6} \mathrm{H}_{4}\right)$ & 10 & - \\
\hline$V-c$ & $\mathrm{O}\left(\mathrm{o}-\mathrm{ClC}_{6} \mathrm{H}_{4}\right)$ & 10 & - \\
\hline$V-d$ & $\mathrm{O}\left(\mathrm{p}-\mathrm{ClC}_{6} \mathrm{H}_{4}\right)$ & 10 & - \\
\hline $\mathrm{V}-\mathrm{e}$ & $\mathrm{O}\left(\mathrm{p}-\mathrm{BrC}_{6} \mathrm{H}_{4}\right)$ & 10 & - \\
\hline$V-f$ & $\mathrm{O}\left(\mathrm{p}-\mathrm{NO}_{2} \mathrm{C}_{6} \mathrm{H}_{4}\right)$ & 10 & - \\
\hline \multicolumn{2}{|c|}{$\begin{array}{l}\text { Control; 2-(2-furyl)-3-(5-nitro-2-furyl)- } \\
\text { acryl-amide }\end{array}$} & 0.5 & 5 \\
\hline
\end{tabular}




\section{Summary}

1. Two type of 5-nitro-2-furoates, phenylthio-5-nitro-2-furoates (IV) and phenyl5-nitro-2-furoates (V), of which benzen ring was substituted with methyl, halogen or nitro group, were prepared for the study of bacteriostatic activities.

2. Some of (IV), p-chlorophenylthio-5-nitro-2-furoate, p-bromo-phenylthio-5-nitro2-furoate and benzylthio-5-nitro-2-furoate, showed strong inhibitive action on Staphylococcus auret!s $209 p$ in vitro.

3. Phenylthio-5-nitro-2-furoates (IV) were more effective than phenyl-5-nitro-2furoates (V).

\section{Acknowledgements}

The authors wish to express their deep gratitude to Dr. W. KASHrWARA, Ueno Seiyaku Co., for supplying of starting material, 5-nitro-2-furfural and Miss M. HAMAJI and Miss K. NAKAne, Fac. of Agr., Nagoya Univ., for operation of micro-elementary analysis.

\section{References}

1) A. Obatake and T. Matsuda: This Bull, 31, 214-218 (1965).

2) E. Niwa, H. Aoki, H. Tanaka and the late T. TAmura: J. Agr. Chem. Soc. Japan, 41, 135-138 (1967).

3) H. SalKachI: Japan, 1431 (1964), Feb. 14, Appl. Nov. 22, 1960; 2 pp., C.A., 60, 11985 (1964). 